\title{
MRI-Directed Brachytherapy for Cancer of the Uterine Cervix: A Case Report, Review, and Perspective on the Importance of Widespread Use of This Technological Advance in the United States
}

\author{
Hiba Z. Ahmed ${ }^{1}$, Srinivasan Vijayakumar ${ }^{1}$, William N. Duggar ${ }^{1}$, Robert Allbright ${ }^{1}$ \\ 1. Radiation Oncology, University of Mississippi Medical Center, Jackson, USA
}

Corresponding author: Robert Allbright, rallbright@umc.edu

\begin{abstract}
Cervical cancer remains a major health challenge in the United States (US), especially among the low socioeconomic and African American populations. The demographics of Mississippi constitute a relatively high percentage of this high-risk population. External beam radiation therapy (EBRT) combined with concurrent chemotherapy and followed by brachytherapy is the gold standard of treatment for stage IB3 through IVA cervical cancer. Arguably, brachytherapy is the most important component of this treatment process. Patterns of Care studies (PCS) and other more recent studies have shown that brachytherapy cannot be omitted or replaced by conventional or image-guided EBRT. The last decade has witnessed the expanding use of image-guided brachytherapy (IGBT). Studies have established the superiority of IGBT over point-based brachytherapy. MRI is associated with superior soft tissue definition compared with CT and is emerging as the new standard of care. The Gynaecological Groupe Européen de Curiethérapie and the European Society for Radiotherapy and Oncology [(GYN) GEC-ESTRO] have recommended that the dose be prescribed to the high-risk clinical target volume (HR-CTV). This volume includes residual tumor present at the time of brachytherapy, the cervix, and any gray areas seen on the scan. The (GYN) GEC-ESTRO has shown that a dose of $>8500 \mathrm{cGy}$ delivered in $<50$ days results in an approximate $10 \%$ increase in pelvic control (PC), disease-specific survival, and overall survival (OS) compared to historical controls. The normal tissue toxicity is comparable or better than historical controls as well. This dose, while maintaining normal tissue constraints, may only be achievable with a hybrid intracavitary/interstitial (IC/IS) needle device guided by MRI-based targeting.
\end{abstract}

The University of Mississippi Medical Center (UMMC) has initiated an MRI-based cervical brachytherapy program and has treated 18 patients to date; our experience confirms the above findings. In this report, we propose that MRI guidance is necessary and a hybrid IC/IS needle device is required to achieve adequate dose coverages.

Review began 04/13/2021 Review ended 05/28/2021 Published 06/07/2021

\section{() Copyright 2021}

Ahmed et al. This is an open access article distributed under the terms of the Creative Commons Attribution License CC-BY 4.0., which permits unrestricted use, distribution, and reproduction in any medium, provided the original author and source are credited.
Categories: Radiation Oncology, Oncology

Keywords: locally advanced cervical cancer, definitive treatment, radiation therapy, concurrent chemoradiation, hdr (high dose rate) brachytherapy, 3-d brachytherapy, image-guided brachytherapy, mri-based brachytherapy, local control, pelvic control

\section{Introduction}

Cervical cancer is a major medical problem among women, especially women of color and those from lower socioeconomic communities, and the population of the State of Mississippi accounts for a significantly high number of this demographic. The State of Mississippi has the second-highest mortality rate due to cervical cancer in the United States (US), at three per 100,000 women versus two per 100,000 women nationally [1]. Mortality is even higher for African American women in Mississippi, which stands at six per 100,000 women compared to four per 100,000 women for African American women nationally and three per 100,000 women for Caucasian women in Mississippi [1]. This data illustrates the severity of the situation related to cervical cancer among African American women in the State of Mississippi. The outcomes among patients who receive the standard-of-care treatment for this condition are generally good. External beam radiation therapy (EBRT) combined with concurrent chemotherapy and followed by brachytherapy is the standard of care for treating stage IB3 through IVA cervical cancer. The application and technique of brachytherapy is arguably the most important component of this treatment paradigm. Studies have shown that brachytherapy is associated with improved survival outcomes.

Since the 1980s, Patterns of Care studies (PCS) have been published emphasizing that intracavitary (IC) and/or interstitial (IS) radiation therapy is the only treatment-related factor associated with improved pelvic control (PC) and overall survival (OS) in the treatment of cervical cancer [2-7]. The PCS have analyzed and reported on the contemporary practices in the treatment of cervical cancer and identified areas that need 
improvement. The early PCS established that academic or training facilities, i.e. high-volume centers, were associated with better treatment outcomes [2,3]. Across all stages, the four-year risk of recurrence was 56\% when IC brachytherapy was not used compared to $14 \%$ with IC. Delving specifically into outcomes for stage IIIB patients, those treated at larger institutions (defined as facilities treating $>50$ new patients per year) had a four-year PC of $60 \%$ versus $28 \%$ for patients treated at smaller practices [2]. This discrepancy in PC was attributed to higher point A doses via increased use of IC. Of note, 35\% of IIIB patients at small practices did not receive IC versus only $12 \%$ of patients treated at large academic institutions. The four-year risk of pelvic failure for stage IIIB in both large and small institutions was $87 \%$ when IC was not used. Even when IC was used, only $37 \%$ of IIIB patients had poor outcomes at large institutions compared to $63 \%$ at small facilities, further emphasizing the importance of physician training, expertise, and patient volume for patient outcomes independent of IC use.

In the early 2000s, there was a trend to replace IC with advanced external beam techniques, such as intensity-modulated radiation therapy (IMRT) and stereotactic body radiation therapy (SBRT), to achieve a similar dose. Han et al. utilized the Surveillance, Epidemiology, and End Results (SEER) database to illustrate that patients with stage IB2-IVA cervical cancer who received brachytherapy after treatment with EBRT had both higher cause-specific survival (CSS) and OS versus those who did not receive brachytherapy [64.3\% vs. 52.5\% (p<0.001), 58.2\% vs. $46.2 \%$ ( $<<0.001)$, respectively] [8]. Gill et al. obtained similar results using data from the National Cancer Database (NCDB) [9]. Gill et al. were able to demonstrate that the omission of brachytherapy had a greater negative impact on OS than the omission of chemotherapy. Hazard ratios indicated an $86 \%$ higher risk of death with the omission of brachytherapy versus a $61 \%$ increased risk with the omission of chemotherapy [9].

Over the past three decades, EBRT has undergone a significant transformation, rapidly evolving from twodimensional (2D) planning in the early phase to three-dimensional (3D) planning and CT-based imageguided radiation therapy (IGRT), and finally to its current modality of MRI-based IGRT. Brachytherapy has witnessed a similar evolution: from 2D point A-based planning to CT point A planning to CT volumetric planning to MRI volumetric planning, pioneered predominately by the Gynaecological Groupe Européen de Curiethérapie and the European Society for Radiotherapy and Oncology [(GYN) GEC-ESTRO]. MRI-based volumetric planning represents a major advance in physicians' abilities to deliver the high doses needed for superior outcomes while minimizing toxicities, and it is rapidly becoming the gold standard of treatment. This paper aims to highlight the University of Mississippi Medical Center's (UMMC) efforts to improve outcomes for cervical cancer patients in Mississippi by developing a state-of-the-art MRI-directed brachytherapy program for treating the cancer of the uterine cervix. We describe our one-year experience to emphasize the importance of a careful planning process, multidisciplinary team approach, perseverance in perfecting patient flow processes, and, inevitability, the need for a superb medical physics support.

\section{Case Presentation}

We conducted a search of the PubMed database to select articles published between 1990-2020 by using the following keywords: "locally advanced cervical cancer", "definitive treatment", "radiation therapy", "concurrent chemoradiation", "HDR (high dose rate) brachytherapy", "3D brachytherapy", "image-guided brachytherapy", "MRI-based brachytherapy", "local control", "pelvic control", and "survival outcomes".

The results of the implementation of UMMC's MRI-based brachytherapy program can be exemplified by the case of Ms. CGR. Ms. CGR was a 47-year-old female patient diagnosed with stage IIIB cervical cancer and had been referred to UMMC for the brachytherapy portion of her treatment. She had received the EBRT portion of her concurrent chemoradiation to $45 \mathrm{~Gy}$ in 25 fractions and a pelvic sidewall boost of $5.4 \mathrm{~Gy}$ in three fractions with weekly cisplatin at an outside facility from October 26, 2020, to December 11, 2020, over the course of 47 days. Her referring physician had first contacted UMMC on December 10, 2020, to discuss Ms. CGR's case and she was seen for a consult by Radiation Oncology on December 15, 2020, the 51st day since the start of her treatment. She had already received a D90 of $4670 \mathrm{cGy}$ to the high-risk clinical target volume (HR-CTV) from her EBRT. At UMMC, she was treated with four fractions of MRI-based high dose rate (HDR) brachytherapy using a hybrid IC/IS technique, subsequently achieving a final HR-CTV D90 of 8669 in 2-Gy-equivalent fractions (EQD2) cGy.

At UMMC, the Department of Radiation Oncology in concert with the Departments of Gynecologic Oncology and Radiology has initiated a protocol utilizing MRI planning for each insertion. Images gathered include the T2-weighted non-contrast para-axial, para-sagittal, and para-coronal sequences, a diffusion sequence for lymph node evaluation, and a straight axial 3D space sequence to facilitate contouring by utilizing a Siemens Aera 1.5 Tesla MRI machine (Siemens Medical Solutions Inc. Malvern, PA). At our institution, the device utilized is primarily the Venezia ${ }^{\mathrm{TM}}$ (Elekta’s Advanced Gynecological Applicator), a hybrid IC/IS instrument (Elekta Inc., Atlanta, GA). The Venezia ${ }^{\mathrm{TM}}$ applicator is utilized without needles for every first fraction, thus serving as a planning template for the use of IS needles during further implants. When IS needles are utilized, up to $10-20 \%$ of the total dose is delivered through the needles [10]. The planning system utilized is Oncentra ${ }^{\circledR}$ Brachy (Elekta Inc., Veenendaal, Netherlands). Ultrasound guidance is used at each insertion. MR images are reviewed with a board-certified radiologist for every patient's, at a minimum, first fraction for tumor/target delineation. The same protocol was used for this patient. Her first insertion used only a tandem with semi-lunar ovoids (T\&O) (Figure 1). 


\section{Cureus}

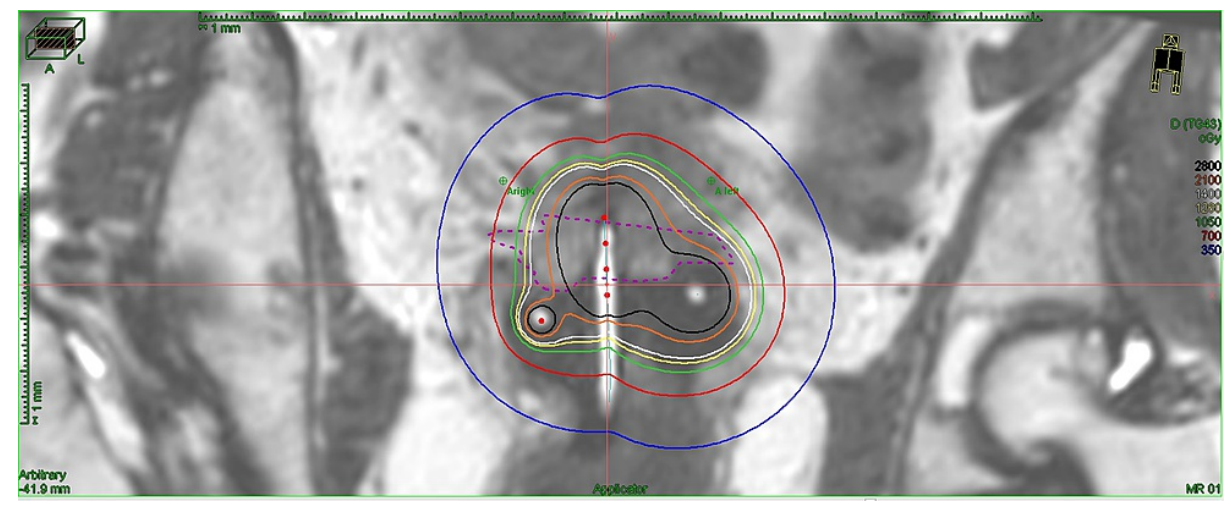

FIGURE 1: Dose profile for the first fraction on MRI using an IC approach alone

MRI: magnetic resonance imaging; IC: intracavitary

This IC-alone approach was only able to deliver 560 cGy to the HR-CTV D90, which equated to 728 cGy EQD2. If an IC approach alone was to be continued, the projected total dose for four fractions of brachytherapy to the HR-CTV D90 from the EBRT and brachytherapy combined would only amount to 7582 cGy (Table 1).

Planning goals and scorecard

$\begin{array}{llllr}\text { Metric } & \text { Primary } & \text { Secondary } & \text { Projected total } & \text { Actual total } \\ \text { Point A mean > } & 6500 & & 8649 \text { cGy } & 5665 \text { cGy } \\ \text { HR-CTV D98 }> & 7500 & & 6537 \text { cGy } & 5137 \text { cGy } \\ \text { HR-CTV D90 } & 9000 & 8500 & 7582 \text { cGy } & 5398 \text { cGy } \\ \text { Bladder D2cc } & 8000 & 9000 & 7042 \text { cGy } & 5158 \text { cGy } \\ \text { Rectum D2cc } & 6500 & 7500 & 7042 \text { cGy } & 5158 \text { cGy } \\ \text { Sigmoid D2cc } & 7000 & 7500 & 6514 \text { cGy } & 5026 \text { cGy } \\ \text { IR-CTV D98 } & 6000 & 6133 \text { cGy } & 5036 \text { cGy }\end{array}$

\section{TABLE 1: Projected and actual total doses after the first fraction of brachytherapy*}

*Outlining the actual total dose to various target volumes and OAR in EQD2 after the first brachytherapy insertion using an IC approach alone and the projected total dose assuming similar doses for the subsequent insertions

HR-CTV: high-risk clinical target volume; D2cc: dose to 2 cc; IR-CTV: intermediate-risk clinical target volume; cGy: centigray; OAR: organs at risk; EQD2: equivalent dose in 2-Gy fractions; IC: intracavitary 


\section{Cureus}

\begin{tabular}{|c|c|c|c|}
\hline Metric & Primary & Secondary & Projected total \\
\hline HR-CTV D90 > & 9000 & 8500 & 8637 cGy \\
\hline Bladder D2cc < & 8000 & 9000 & 8050 cGy \\
\hline Rectum D2cc < & 6500 & 1500 & 8160 cGy \\
\hline
\end{tabular}

\section{TABLE 2: Projected total doses with IC-alone approach with $\sim 700$ cGy per fraction*}

*Table estimating the total dose to the HR-CTV D90, bladder D2cc, and rectum D2cc in EQD2 assuming each fraction was able to achieve an HRCTV D90 of 700 cGy with an IC-alone approach (i.e., assuming 700 cGy was deliverable to the HR-CTV D90 without the use of needles)

Due to the inability to deliver a sufficient dose while respecting the organs at risk (OAR) through an IC approach alone, the remaining three insertions were done using a hybrid IC/IS instrument (Figures 2, 3).

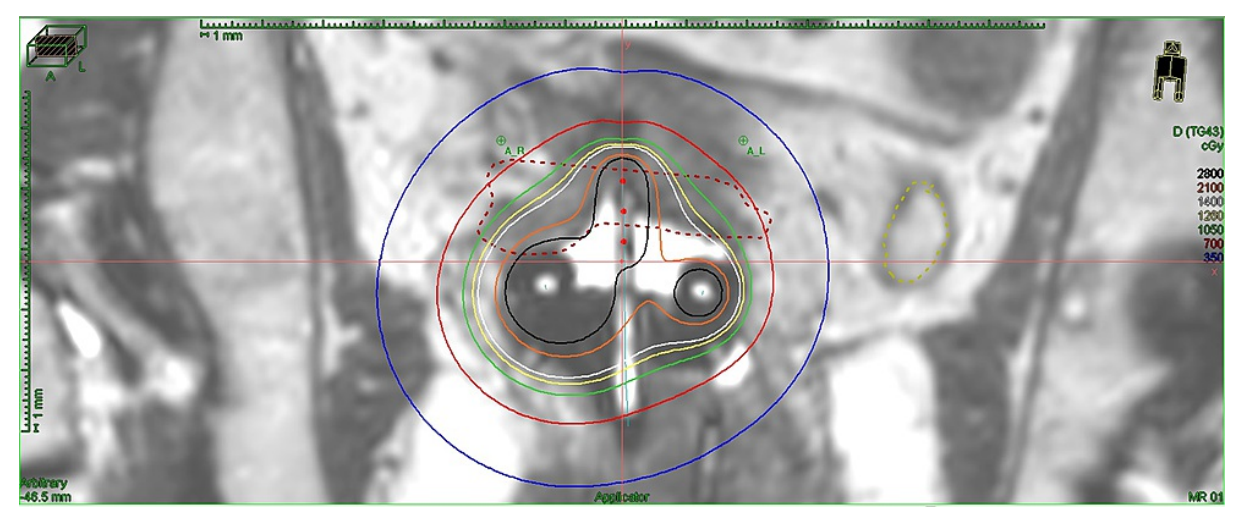

FIGURE 2: Dose profile of one of the fractions on MRI using an IC/IS approach illustrating the tandem and needles

MRI: magnetic resonance imaging; IC/IS: intracavitary/interstitial

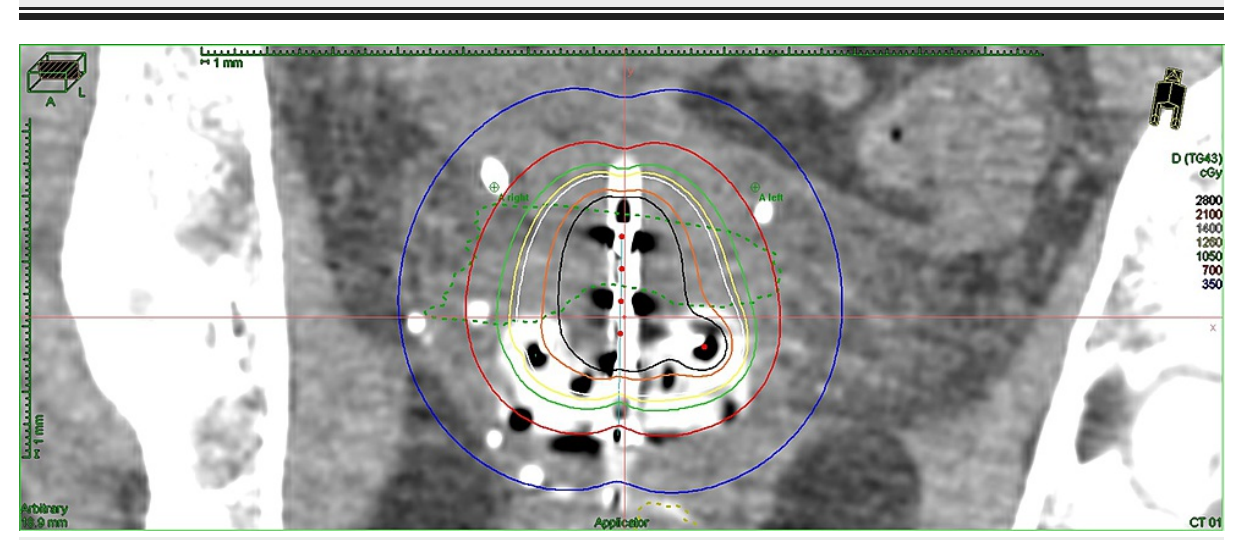

FIGURE 3: Dose profile of one of the fractions using an IC/IS approach on the planning CT scan illustrating the tandem and needles

IC/IS: intracavitary/interstitial; CT: computed tomography

The subsequent three fractions each delivered a dose over 700 cGy, converted to 1094 cGy, 1083 cGy, and 1094 cGy in EQD2 (Table 3). 


\section{Cureus}

\begin{tabular}{|c|c|c|c|c|}
\hline Fraction \# & One & Two & Three & Four \\
\hline Fraction date & $12 / 22 / 20$ & $12 / 29 / 20$ & $1 / 5 / 20$ & $1 / 12 / 21$ \\
\hline Point A mean & 995 cGy & 600 cGy & 608 cGy & 773 cGy \\
\hline HR-CTV D98 & 467 cGy & 746 cGy & 804 cGy & 728 cGy \\
\hline HR-CTV D90 & 992 cGy & 992 cGy & 992 cGy & 992 cGy \\
\hline Bladder D2cc & 628 cGy & 800 cGy & $1050 \mathrm{cGy}$ & 963 cGy \\
\hline Rectum D2cc & 628 cGy & 749 cGy & 724 cGy & 651 cGy \\
\hline Sigmoid D2cc & 496 cGy & 749 cGy & 724 cGy & \\
\hline RV point dose & 470 cGy & 360 cGy & 430 cGy & 420 cGy \\
\hline IR-CTV D98 & 366 cGy & 352 cGy & 338 cGy & $437 \mathrm{cGy}$ \\
\hline
\end{tabular}

\section{TABLE 3: Doses to target volumes and OAR*}

*Table listing the actual doses delivered to various target volumes and OAR for each fraction of brachytherapy in EQD2

HR-CTV: high-risk clinical target volume; D2cc: dose to $2 \mathrm{cc}$; RV: rectovaginal; IR-CTV: intermediate-risk clinical target volume; cGy: centigray; OAR: organs at risk; EQD2: equivalent dose in 2-Gy fractions

Through this method, the patient was able to receive a total dose of 8669 cGy, surpassing the minimum dose recommendation of $>8500 \mathrm{cGy}$ while still meeting OAR dose constraints, specifically with a rectal D2cc dose of $7282 \mathrm{cGy}$, well below the planning goal of $<7500$ cGy (Table 4).

Planning goals and scorecard

$\begin{array}{lllll}\text { Metric } & \text { Primary } & \text { Secondary } & \text { Projected total } & \text { Actual total } \\ \text { Point A mean }> & 6500 & & 7646 \text { cGy } & 7646 \text { cGy } \\ \text { HR-CTV D98 }> & 7500 & & 7414 \text { cGy } & 7414 \text { cGy } \\ \text { HR-CTV D90 }> & 9000 & 8500 & 8637 \text { cGy } & 8637 \text { cGy } \\ \text { Bladder D2cc }< & 8000 & 9000 & 7972 \text { cGy } & 7972 \text { cGy } \\ \text { Rectum D2cc }< & 6500 & 7500 & 7282 \text { cGy } & 7282 \text { cGy } \\ \text { Sigmoid D2cc }< & 7000 & 7500 & 7155 \text { cGy } & 6499 \text { cGy } \\ \text { RV point dose }< & 6500 & 7500 & 6910 \text { cGy } & 6910 \text { cGy } \\ \text { IR-CTV D98 }> & 6000 & & 6163 \text { cGy } & 6163 \text { cGy }\end{array}$

\section{TABLE 4: Projected and actual dose after the fourth fraction of brachytherapy*}

*Table outlining the actual total dose to various target volumes and OAR in EQD2 after the fourth, final brachytherapy insertion (one IC-alone and three IC/IS)

HR-CTV: high risk-clinical target volume; D2cc: dose to 2 cc; RV: rectovaginal; IR-CTV: intermediate-risk clinical target volume; cGy: centigray; OAR: organs at risk; EQD2: equivalent dose in 2-Gy fractions; IC/IS: intracavitary/interstitial

\section{Discussion}

The case detailed above illustrates many salient points regarding the necessity of employing state-of-the-art MRI-directed brachytherapy in the treatment of cancer of the uterine cervix in a rural state, such as Mississippi. At UMMC, the Department of Radiation Oncology in concert with the Departments of Gynecologic Oncology and Radiology has initiated a protocol utilizing MRI planning for each insertion. Thus far, 18 cervical cancer patients have received MRI-based brachytherapy at UMMC. The average HR-CTV 
volume has been $28.8 \mathrm{~cm}^{3}$, and $28 \%$ of the cases have required needles (Table 5).

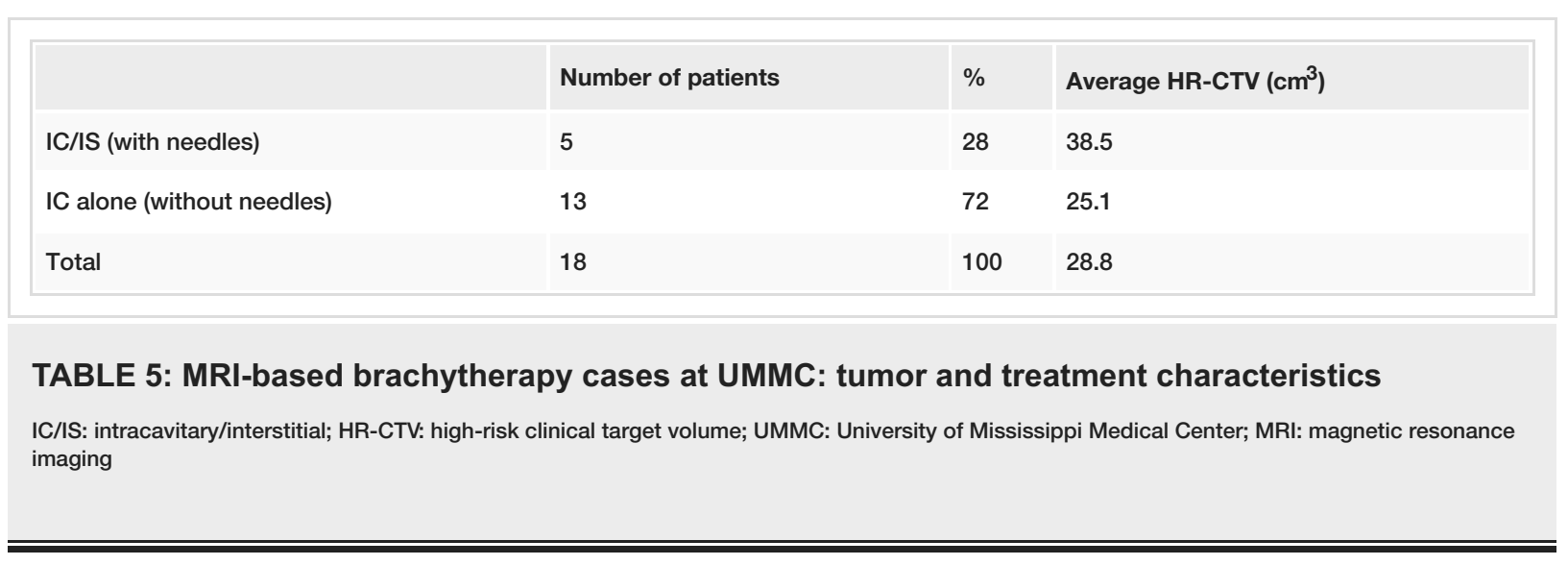

We have drawn the following conclusions from our experiences:

1. UMMC, the state's only academic medical center, is the only institution capable of providing MRI-based brachytherapy for this disease currently. Many smaller radiation oncology facilities that are capable of providing EBRT in the state do not have the infrastructure to perform even non-MRI-based brachytherapy. Treating patients who have received their EBRT portion of treatment at an outside facility requires significant medical physics support to obtain Digital Imaging and Communications in Medicine (DICOM)based data and composite those radiation therapy plans with brachytherapy dose profiles.

2. If we did not have the ability to perform MRI-based brachytherapy, our treatment volumes might have missed areas of viable disease that would not have been apparent on more traditional imaging.

3. If we did not have the hybrid IC/IS brachytherapy applicator, we would have failed to administer sufficient doses to the high-risk volume without exceeding the tolerances of normal tissues.

We will expand further on the above-listed points here.

The evolution of brachytherapy from 2D to 3D-based planning has been illustrated by the Soutien aux Techniques Innovantes et Coûteuses (STIC) Study from France. The STIC Study was a prospective, multicentric trial designed to compare local/locoregional control outcomes in patients treated with either a 2D or 3D (mostly CT but some MRI) brachytherapy plan [10]. Specifically, patients treated with 3D brachytherapy had better local relapse-free survival $(78.5 \%$ vs. $73.9 \%$; $\mathrm{p}=0.003)$ and locoregional relapse-free survival (69.6\% vs. $61.2 \%$; $p=0.001$ ) than the 2D cohort. Patients in the 3D cohort developed fewer grade $3-4$ toxicities (2.6\% vs. $22.7 \%$; $\mathrm{p}=0.002)$ [10]. This provides strong evidence that 3D-based dosimetric planning is superior to 2D-based dosimetric planning for brachytherapy.

MRI is associated with superior soft tissue delineation and resolution compared to CT. Moreover, MRI provides a more detailed image of the parametrium and residual disease. Viswanathan et al. prospectively compared dose-volume histograms (DVHs) for the targets and OARs with CT vs. MRI 3D planning [11]. The authors utilized a standardized CT contouring protocol intended to approximate the corresponding (GYN) GEC-ESTRO MRI contouring volumes. The CT volumes consistently overestimated the width of the tumor. MRI-based planning resulted in a greater volume of the HR-CTV treated to the prescription dose or higher. MRI yielded a D100 of $96 \%$ versus $86 \%$ with CT $(\mathrm{p}=0.01)$ [11].

In the early 2000s, (GYN) GEC-ESTRO published recommendations for the acquisition of MRI images to utilize in image-guided brachytherapy (IGBT) [12,13]. The protocol employs T2-weighted non-contrast images of the pelvis. Specific orientations captured include para-axial, para=sagittal, and para-coronal [12]. (GYN) GEC-ESTRO also formulated a series of definitions for the generation of target and OAR DVHs [13]. Gross tumor volume (GTV) has been defined as tumor present at the time of brachytherapy as represented by high signal intensity mass(es) (FSE, T2). The HR-CTV includes the entire cervix, the extra-cervical tumor extension, and any "gray" areas presumed to be related to the disease. The intermediate-risk clinical target volume (IR-CTV) is defined as the HR-CTV plus a $0.5-1.5-\mathrm{cm}$ expansion excluding the bladder and rectum or the original extent of the tumor. Dose recommendations for the D90 HR-CTV, D98 HR-CTV, D98 GTV, D98 IR-CTV, and point A in EQD2 are >85 Gy, >75 Gy, >90 Gy, >60 Gy, and >65 Gy, respectively (Table ๑). 


\section{Cureus}

\begin{tabular}{|l|l|l|l|l|l|}
\hline & D90 HR-CTV EQD2 & D98 HR-CTV EQD2 & D98 GTV EQD2 & D98 IR-CTV EQD2 & Point A EQD2 \\
\hline Ideal planning goal & $>90 \mathrm{~Gy},<95 \mathrm{~Gy}$ & $>75 \mathrm{~Gy}$ & $>95 \mathrm{~Gy}$ & $>60 \mathrm{~Gy}$ & $>65$ Gy \\
\hline Recommended minimum & $>85 \mathrm{~Gy}$ & & $>90 \mathrm{~Gy}$ & & \\
\hline
\end{tabular}

\section{TABLE 6: (GYN) GEC-ESTRO planning target goals}

HR-CTV: high-risk clinical target volume; EQD2: equivalent dose in 2-Gy fractions; GTV: gross tumor volume; (GYN) GEC-ESTRO: Gynaecological Groupe Européen de Curiethérapie and the European Society for Radiotherapy and Oncology

Dose constraints for the dose to $2 \mathrm{cc}$ (D2cc) of the bladder, rectum, and sigmoid in EQD2 are <90 Gy, <75 Gy, and $<75 \mathrm{~Gy}$, respectively (Table 7). A rectovaginal (RV) point was defined as a point $5 \mathrm{~mm}$ posterior to the vaginal mucosa at the center of the sources [14]. This serves as a surrogate for estimating late fibrosis of the vagina. EQD2 should ideally be $<65$ Gy with a secondary acceptable upper limit of $<75$ Gy as well (Table 7).

\begin{tabular}{|c|c|c|c|c|}
\hline & Bladder D2cc EQD2 & Rectum D2cc EQD2 & Sigmoid D2cc EQD2 & RV point dose \\
\hline Ideal planning goal & $<80$ Gy & $<65$ Gy & $<70$ Gy & $<65$ Gy \\
\hline Secondary acceptable maximum & $<90$ Gy & $<75$ Gy & $<75$ Gy & $<75$ Gy \\
\hline
\end{tabular}

\section{TABLE 7: (GYN) GEC-ESTRO OAR planning goals}

D2cc: dose to 2 cc; EQD2: equivalent dose in 2-Gy fractions; RV: rectovaginal; (GYN) (GYN) GEC-ESTRO: Gynaecological Groupe Européen de Curiethérapie and the European Society for Radiotherapy and Oncology; OAR: organs at risk

The (GYN) GEC-ESTRO recommendations listed above were generated from data gathered from a series of sentinel studies as part of a multicenter international study of MRI-based brachytherapy starting in 2008. The first of these studies was image-guided intensity-modulated external beam radiochemotherapy and MRI-guided adaptive brachytherapy in locally advanced cervical cancer (EMBRACE I), which was a prospective single-arm study consisting of patients diagnosed with Fédération Internationale de Gynécologie et d'Obstétrique (FIGO) IB-IVA biopsy-proven squamous cell carcinoma (SCC), adenocarcinoma (AC), or adenosquamous carcinoma (AdSq) of the cervix [15]. Patients were treated with concurrent chemoradiation and MRI-based IGBT [15]. The study aimed to demonstrate superior clinical outcomes of local control (LC), survival, morbidity, and quality of life (QoL) for MRI-based IGBT. This study had accrued 1,416 patients by 2015 . Vide infra for results.

Simultaneously, (GYN) GEC-ESTRO initiated a retrospective analysis of patients treated with MRI-based IGBT prior to the initiation of EMBRACE I in 2008; this was the RetroEMBRACE [15]. RetroEMBRACE had three main foci of investigation [16-18]. The first was to illustrate that IGBT combined with concurrent chemoradiation yields improved LC, PC, OS, and CSS compared to 2D brachytherapy historical controls [17]. Secondly, they sought to establish optimal overall treatment time and dose minimums to target volumes [18]. The last goal was to demonstrate that an IC/IS hybrid approach is superior to an IC approach alone for increasing treatment volumes [16]. Vide infra for results.

In 2016, EMBRACE II, another prospective single-arm study, was initiated, which is still undergoing [15]. EMBRACE II represented a further refinement in brachytherapy techniques as advanced by EMBRACE I and RetroEMBRACE. EMBRACE II will attempt to increase QoL by decreasing vaginal fibrosis [19]. One way to achieve this would be to change the weighting of the tandem-to-ring ratio from 50:50 to 66:33. EMBRACE II will also advocate for the increased use of the hybrid IC/IS devices with IS needles, thereby increasing the doses to large tumors and decreasing the ring contribution, which will, in turn, decrease the dose to the RV point. EMBRACE II will also aim to decrease the dose to the vagina via the external beam component by decreasing the overall EBRT volume through "reduced PTV margins, appropriate target selection, systemic contouring, and meticulous treatment planning for IMRT/volume metric modeled arc therapy (VMAT)" [19]. By implementing these changes, it may be theoretically possible to decrease the risk of vaginal stenosis from $21 \%$ to $14 \%[19]$.

The bulk of the data regarding MRI-based brachytherapy stems from EMBRACE I and RetroEMBRACE. The following data is predominately from RetroEMBRACE. When treated to a dose of at least $85 \mathrm{~Gy}$ (D90) delivered in seven weeks, tumors limited in size to $20 \mathrm{~cm}^{3}$ had a three-year LC of 94\% [18]. Intermediatesized tumors with volumes between $20-30 \mathrm{~cm}^{3}$ had a three-year LC of $93 \%$. Tumors with volumes $31-70 \mathrm{~cm}^{3}$ 
had a three-year LC of $86 \%$. The overall LC was $91 \%$. The mean D 90 for the HR-CTV using an IC approach alone was 83 Gy versus $92 \mathrm{~Gy}$ for an IC/IS approach ( $p<0.01$ ). For patients with tumor volumes $>30 \mathrm{~cm}^{3}$, an IC/IS approach provided a 10\% absolute increase in three-year LC [16]. From this data, they were able to extrapolate that for every $10 \mathrm{~cm}^{3}$-increase in the volume of tumor size $>30 \mathrm{~cm}^{3}$, an increase of $5 \mathrm{~Gy}$ to the HR-CTV would be required to achieve equivalent levels of LC [18]. Similarly, they were able to extrapolate that for every one-week increase in the overall treatment period beyond seven weeks, again an increase of 5 Gy to the HR-CTV would be required to compensate for that delay in treatment. The three-year PC was $98 \%$ for stage IB, 93\% for stage IIB, and 79\% for stage IIIB [20]. Overall, crude PC was $87 \%$ [17]. The overall risk of nodal failure was $11 \%$ [21]. Patients who were initially node-positive at diagnosis were more likely to develop nodal failure at $16 \%$ versus patients who were initially node-negative at diagnosis, who had a nodal failure of $7 \%$. Nodal failures most commonly occurred at the superior pelvic field border and in the para-aortic region [21]. EMBRACE II will address nodal failure by boosting nodes that are positive at presentation with simultaneous integrated boost (SIB) technique to approximately 60 Gy [19]. EMBRACE II will also divide EBRT patients into small pelvic, large pelvic, and pelvic and para-aortic fields based on varying degrees of nodal positivity at presentation. The actuarial five-year OS for the entire RetroEMBRACE cohort was $65 \%$ and five-year CSS was 73\% (Table 8) [17].

\begin{tabular}{|c|c|c|c|}
\hline & Five-year PC & Five-year CSS & Five-year OS \\
\hline RetroEMBRACE & $84 \%$ & $73 \%$ & $65 \%$ \\
\hline EMBRACE I & $91 \%^{*}$ & Not reported & Not reported \\
\hline Vale meta-analysis & $77 \%$ & $58 \% \%^{\star x}$ & $66 \%$ \\
\hline UK meta-analysis & $78 \%$ & $59 \%$ & $55 \%$ \\
\hline NCDB & Not reported & Not reported & $54 \%$ \\
\hline SEER & Not reported & $64.3 \%{ }^{\star \star \star}$ & $54 \%$ \\
\hline \multicolumn{4}{|c|}{${ }^{\star}$ Three-year LC. ${ }^{\star *}$ Five-year DFS. ${ }^{* \star *}$ Four-year CSS } \\
\hline \multicolumn{4}{|c|}{$\begin{array}{l}\text { EMBRACE: international study on MRI-guided brachytherapy in locally advanced cervical cancer; NCDB: National Cancer Database; SEER: } \\
\text { Surveillance, Epidemiology, and End Results; MRI: magnetic resonance imaging; PC: pelvic control; CSS: cause-specific survival; OS: overal } \\
\text { survival; LC: local control; DFS: disease-free survival }\end{array}$} \\
\hline
\end{tabular}

Historical controls for patients treated with chemoradiation with 2D brachytherapy include the Vale metaanalysis, the United Kingdom (UK) meta-analysis, SEER, and NCDB data $[8,9,22,23]$. The Vale meta-analysis analyzed 13 randomized trials worldwide [22]. This data suggested that concurrent chemoradiation improved OS by $6 \%$ at five years. The benefit was greater in stage IA and IIA disease than IIIA and IVA disease. PC at five years in RetroEMBRACE was $84 \%$ compared to Vale's $77 \%$ (Table 7) [17,23]. The OS for the RetroEMBRACE cohort was $65 \%$ at five years [17]. The UK and Vale meta-analyses five-year OS were $55 \%$ and $66 \%$, respectively [22,23]. The SEER and NCDB five-year OS were $54[8,9]$. The UK five-year CSS was $59 \%$ [23]. The above data indicate a $\sim 10 \%$ increase in PC, OS, and CSS for RetroEMBRACE over historical controls.

The American experience with 3D MRI-based brachytherapy is best represented by the University of Pittsburgh study, which accrued patients from 2007 to 2018 [24]. A total of 239 women were studied. The median HR-CTV D 90 was $83.7 \mathrm{~Gy}$ and the volume was $31 \mathrm{~cm}^{3}$. Treatment time averaged 51 days. LC at five years was $90.8 \%$ and decreased with AC and HR-CTV $>40 \mathrm{~cm}^{3}$. Two-year progression-free survival (PFS) was $84.1 \%$ for tumors $<40 \mathrm{~cm}^{3}$ and $66.2 \%$ for tumors $>40 \mathrm{~cm}^{3}$. Two-year OS for tumors $<40 \mathrm{~cm}^{3}$ was $90.4 \%$ while survival for tumors $>40 \mathrm{~cm}^{3}$ was $65 \%$. Patients with AC, poor response to EBRT, or HR-CTV $>40 \mathrm{~cm}^{3}$ had inferior LC independent of any increase in doses above $85 \mathrm{~Gy}$. An IC/IS hybrid device was used in $38.9 \%$ of the cases [24].

In 2020, The American Society for Radiation Oncology (ASTRO) issued guidelines for the treatment of cervical cancer, including brachytherapy [25]. The recommendations were divided into strong and conditional. ASTRO strongly recommended intra-procedure imaging, e.g., ultrasound, for device placement and MR or CT-based volumetric planning [10,17,25-32]. If this was unavailable, 2D point-based planning was strongly recommended [25,33-37]. ASTRO also strongly recommended total EQD2 of brachytherapy and EBRT to $>80 \mathrm{~Gy}[13,25,30,38]$. A D90 $\geqslant 8500 \mathrm{cGy}$ was conditionally recommended for tumors that poorly responded to EBRT or tumors $>4 \mathrm{~cm}$, with careful consideration to normal tissue constraints [18,39-41]. 
Also, ASTRO conditionally recommended the utilization of a hybrid IC/IS device to improve dose distribution and to respect OAR constraints. Additionally, ASTRO strongly recommended the use of volumetric planning for OAR, but if it was not available, it strongly recommended 2D point-based constraints [17,25,31,33,34,36,37,40,42-44]. Ideal dose constraints for D2cc of the rectum, bladder, RV point, sigmoid, and bowel in EQD2 were $<65 \mathrm{~Gy},<80 \mathrm{~Gy},<65 \mathrm{~Gy}$ (point dose), <70 Gy and <70 Gy, respectively with additional maximum respective dose constraints of: $<75,<90,<75$ (point dose), $<75$, and $<75$ Gy, respectively (Table 9).

\begin{tabular}{|c|c|c|c|c|c|}
\hline & $\begin{array}{l}\text { Bladder D2cc } \\
\text { EQD2 }\end{array}$ & $\begin{array}{l}\text { Rectum D2cc } \\
\text { EQD2 }\end{array}$ & $\begin{array}{l}\text { Sigmoid D2cc } \\
\text { EQD2 }\end{array}$ & $\begin{array}{l}\text { Bowel D2cc } \\
\text { EQD2 }\end{array}$ & $\begin{array}{l}\text { RV point } \\
\text { dose }\end{array}$ \\
\hline Ideal planning goal & $<80$ Gy & $<65$ Gy & $<70$ Gy & $<70$ Gy & $<65$ Gy \\
\hline $\begin{array}{l}\text { Secondary acceptable } \\
\text { maximum }\end{array}$ & $<90$ Gy & $<75$ Gy & $<75$ Gy & <75 Gy & $<75$ Gy \\
\hline
\end{tabular}

\section{TABLE 9: ASTRO OAR dose constraints}

D2cc: dose to 2 cc; EQD2: equivalent dose in 2-Gy fractions; RV: rectovaginal; ASTRO: American Society for Radiation Oncology; OAR: organs at risk

The risk for vaginal stenosis in EMBRACE I was found to be $20 \%$ at $65 \mathrm{~Gy}, 27 \%$ at $75 \mathrm{~Gy}$, and $34 \%$ at 85 Gy [44]. EMBRACE I also demonstrated a risk of rectovaginal fistula formation at three years of $12.5 \%$ with a D2 $\mathrm{cm}^{3}$ with doses $\geqslant 75$ Gy Gy versus a risk of $<2.7 \%$ with D2 $\mathrm{cm}^{3}$ doses $<75$ Gy $[40]$.

These recommendations are very broad and are intended to be inclusive of practices/institutions with varying degrees of technology. Some practices do not have access to an MRI for treatment planning or possess an IC/IS device. Without IC/IS, it may be very difficult to achieve a dose of $85 \mathrm{~Gy}$ with increasing tumor volumes. At EMBRACE institutions that primarily use an IC technique, half of the patients with HR-

CTV volumes $>30 \mathrm{~cm}^{3}$ received a D90 EQD2 dose of $<85 \mathrm{~Gy}$ [19]. The $80 \mathrm{~Gy}$ threshold strongly recommended by ASTRO reflects the results of a trial published by Rao et al., which included 80 patients with stage IIB and IIIB cervical cancer [38]. Patients received 46 Gy EBRT followed by a brachytherapy boost of 600 cGy times four or 800 cGy times three. This resulted in a total EQD2 of 78 or 82 Gy, respectively. They found no significant difference between the two treatment groups. At 30 months, both LC and disease-free survival (DFS) were between $80-90 \%$ [38].

We feel that the ASTRO guidelines could have been stronger, which could have helped push policy changes to promote more widespread use of MRI-based brachytherapy for cancer of the cervix. The current bottleneck preventing a more universal use of MRI-based brachytherapy is the lack of investment by even major medical centers. Recommendations made by major cancer policy-making organizations such as the National Cancer Institute (NCI), ASTRO, American Society of Clinical Oncology (ASCO), and American College of Surgeons Commission on Cancer (ACS CoC) can lead to more investment in infrastructure by healthcare facilities and will improve the overall outcomes in cancer of the cervix in the future. Insurance providers also need to consider offering coverage for referral services to MRI-based brachytherapy-capable facilities similar to referrals to proton beam facilities for children with cancer and patients diagnosed with rare tumors such as chordoma of the clivus.

The department has medical physicists who specialize in brachytherapy, and their assistance enables us to perform these complex procedures, as well as the support our institution. The implementation of MRI-based gynecological brachytherapy was planned before its outset and involved multiple disciplines including Gynecologic Oncology, Diagnostic Radiology, Anesthesiology, and Medical Physics. Operational Administration coordinates the patient flow, DICOM image transfers, and the care, safety, and convenience of patients and their families.

In a very recent publication, the risk for vaginal stenosis from EMBRACE I was reported to be $20 \%$ at $65 \mathrm{~Gy}$, $27 \%$ at $75 \mathrm{~Gy}$, and $34 \%$ at $85 \mathrm{~Gy}$ [44]. EMBRACE I also demonstrated a risk of RV fistula formation at three years of $12.5 \%$ with a D2 $\mathrm{cm}^{3}$ with doses $\geqslant 75$ Gy versus a risk of $<2.7 \%$ with D2 $\mathrm{cm}^{3}$ doses $<75$ Gy [40]. Most recently, EMBRACE I published its results on risk factors and dose effects for bladder fistula and cystitis. They found that the crude incidence rate for bladder fistula greater than grade 1 was $0.7 \%$ and that an increase from $75 \mathrm{~Gy}$ to $80 \mathrm{~Gy}$ in bladder D2 $\mathrm{cm}^{3}$ resulted in an increase from $8 \%$ to $13 \%$ of cystitis greater than grade 1 [45].

\section{Conclusions}


In this report, we highlighted the importance of using MRI-based brachytherapy in the treatment of cancer of the uterine cervix to improve outcomes and the necessity of adopting a team approach in implementing and operationalizing such a program. The benefits of such a program can enable the delivery of adequate doses to the tumor volumes without exceeding the dose tolerances of the normal tissues. Finally, we cannot overemphasize the need for a careful multidisciplinary approach to carry out this complex procedure safely for the benefit of our patients.

\section{Additional Information \\ Disclosures}

Human subjects: Consent was obtained or waived by all participants in this study. University of Mississippi Medical Center Institutional Review Board issued approval 2012-0147. This study has been approved by the University of Mississippi Medical Center Institutional Review Board. Conflicts of interest: In compliance with the ICMJE uniform disclosure form, all authors declare the following: Payment/services info: All authors have declared that no financial support was received from any organization for the submitted work. Financial relationships: All authors have declared that they have no financial relationships at present or within the previous three years with any organizations that might have an interest in the submitted work. Other relationships: All authors have declared that there are no other relationships or activities that could appear to have influenced the submitted work.

\section{References}

1. NIH: State Cancer Profiles. (2018). Accessed: January 24, 2020: https://statecancerprofiles.cancer.gov/.

2. Hanks GE, Herring DF, Kramer S: Patterns of care outcome studies. Results of the national practice in cancer of the cervix. Cancer. 1983, 51:959-67. 10.1002/1097-0142(19830301)51:5<959::aidcncr2820510533>3.0.co;2-k

3. Lanciano RM, Won M, Coia LR, Hanks GE: Pretreatment and treatment factors associated with improved outcome in squamous cell carcinoma of the uterine cervix: a final report of the 1973 and 1978 patterns of care studies. Int J Radiat Oncol Biol Phys. 1991, 20:667-76. 10.1016/0360-3016(91)90007-q

4. Eifel PJ, Moughan J, Owen J, Katz A, Mahon I, Hanks GE: Patterns of radiotherapy practice for patients with squamous carcinoma of the uterine cervix: patterns of care study. Int J Radiat Oncol Biol Phys. 1999, 43:3518. 10.1016/s0360-3016(98)00401-5

5. Eifel PJ, Moughan J, Erickson B, Iarocci T, Grant D, Owen J: Patterns of radiotherapy practice for patients with carcinoma of the uterine cervix: a patterns of care study. Int J Radiat Oncol Biol Phys. 2004, 60:114453. 10.1016/j.ijrobp.2004.04.063

6. Erickson B, Eifel P, Moughan J, Rownd J, Iarocci T, Owen J: Patterns of brachytherapy practice for patients with carcinoma of the cervix (1996-1999): a patterns of care study. Int J Radiat Oncol Biol Phys. 2005, 63:1083-92. 10.1016/j.ijrobp.2005.04.035

7. Eifel PJ, Ho A, Khalid N, Erickson B, Owen J: Patterns of radiation therapy practice for patients treated for intact cervical cancer in 2005 to 2007: a quality research in radiation oncology study. Int J Radiat Oncol Biol Phys. 2014, 89:249-56. 10.1016/j.ijrobp.2013.11.228

8. Han K, Milosevic M, Fyles A, Pintilie M, Viswanathan AN: Trends in the utilization of brachytherapy in cervical cancer in the United States. Int J Radiat Oncol Biol Phys. 2013, 87:111-9. 10.1016/j.ijrobp.2013.05.033

9. Gill BS, Lin JF, Krivak TC, et al.: National Cancer Data Base analysis of radiation therapy consolidation modality for cervical cancer: the impact of new technological advancements. Int J Radiat Oncol Biol Phys. 2014, 90:1083-90. 10.1016/j.ijrobp.2014.07.017

10. Charra-Brunaud C, Harter V, Delannes M, et al.: Impact of 3D image-based PDR brachytherapy on outcome of patients treated for cervix carcinoma in France: results of the French STIC prospective study. Radiother Oncol. 2012, 103:305-13. 10.1016/j.radonc.2012.04.007

11. Viswanathan AN, Dimopoulos J, Kirisits C, Berger D, Pötter R: Computed tomography versus magnetic resonance imaging-based contouring in cervical cancer brachytherapy: results of a prospective trial and preliminary guidelines for standardized contours. Int J Radiat Oncol Biol Phys. 2007, 68:491-8. 10.1016/j.ijrobp.2006.12.021

12. Dimopoulos JC, Petrow P, Tanderup K, et al.: Recommendations from Gynaecological (GYN) GEC-ESTRO Working Group (IV): basic principles and parameters for MR imaging within the frame of image based adaptive cervix cancer brachytherapy. Radiother Oncol. 2012, 103:113-22. 10.1016/j.radonc.2011.12.024

13. Haie-Meder C, Pötter R, Van Limbergen E, et al.: Recommendations from Gynaecological (GYN) GEC-ESTRO Working Group (I): concepts and terms in 3D image based 3D treatment planning in cervix cancer brachytherapy with emphasis on MRI assessment of GTV and CTV. Radiother Oncol. 2005, 74:235-45. 10.1016/j.radonc.2004.12.015

14. No authors listed: Report 89. J ICRU. 2013, 13:1-2. 10.1093/jicru/ndw042

15. EMBRACE studies and EMBRACE research. (2021). Accessed: February 1, 2021: http://www.embracestudy.dk.

16. Fokdal L, Sturdza A, Mazeron R, et al.: Image guided adaptive brachytherapy with combined intracavitary and interstitial technique improves the therapeutic ratio in locally advanced cervical cancer: analysis from the retroEMBRACE study. Radiother Oncol. 2016, 120:434-40. 10.1016/j.radonc.2016.03.020

17. Sturdza A, Pötter R, Fokdal LU, et al.: Image guided brachytherapy in locally advanced cervical cancer: Improved pelvic control and survival in RetroEMBRACE, a multicenter cohort study. Radiother Oncol. 2016, 120:428-33. 10.1016/j.radonc.2016.03.011

18. Tanderup K, Fokdal LU, Sturdza A, et al.: Effect of tumor dose, volume and overall treatment time on local control after radiochemotherapy including MRI guided brachytherapy of locally advanced cervical cancer. 
Radiother Oncol. 2016, 120:441-6. 10.1016/j.radonc.2016.05.014

19. Pötter R, Tanderup K, Kirisits C, et al.: The EMBRACE II study: The outcome and prospect of two decades of evolution within the GEC-ESTRO GYN working group and the EMBRACE studies. Clin Transl Radiat Oncol. 2018, 9:48-60. 10.1016/j.ctro.2018.01.001

20. Tan LT, Tanderup K, Hoskin P, Cooper R, Pötter R: Image-guided adaptive brachytherapy for cervix cancer a story of successful collaboration within the GEC-ESTRO GYN network and the EMBRACE studies. Clin Oncol (R Coll Radiol). 2018, 30:397-9. 10.1016/j.clon.2018.04.005

21. Nomden CN, De Leeuw A, Tanderup K, et al.: Nodal failure after chemoradiation and magnetic resonance imaging guided adaptive BT in cervical cancer: a subanalysis within Embrace. Int J Radiat Oncol Biol Phys. 2016, 96:12. 10.1016/j.ijrobp.2016.06.044

22. Chemoradiotherapy for Cervical Cancer Meta-Analysis Collaboration: Reducing uncertainties about the effects of chemoradiotherapy for cervical cancer: a systematic review and meta-analysis of individual patient data from 18 randomized trials. J Clin Oncol. 2008, 26:5802-12. 10.1200/JCO.2008.16.4368

23. Vale CL, Tierney JF, Davidson SE, Drinkwater KJ, Symonds P: Substantial improvement in UK cervical cancer survival with chemoradiotherapy: results of a Royal College of Radiologists' audit. Clin Oncol (R Coll Radiol). 2010, 22:590-601. 10.1016/j.clon.2010.06.002

24. Horne ZD, Karukonda P, Kalash R, et al.: Single-institution experience in 3D MRI-based brachytherapy for cervical cancer for 239 women: can dose overcome poor response?. Int J Radiat Oncol Biol Phys. 2019, 104:157-64. 10.1016/j.ijrobp.2018.12.042

25. Chino J, Annunziata CM, Beriwal S, et al.: Radiation therapy for cervical cancer: executive summary of an ASTRO clinical practice guideline. Pract Radiat Oncol. 2020, 10:220-34. 10.1016/j.prro.2020.04.002

26. Sapienza LG, Jhingran A, Kollmeier MA, Lin LL, Calsavara VF, Gomes MJL, Baiocchi G: Decrease in uterine perforations with ultrasound image-guided applicator insertion in intracavitary brachytherapy for cervical cancer: a systematic review and meta-analysis. Gynecol Oncol. 2018, 151:573-8. 10.1016/j.ygyno.2018.10.011

27. Castelnau-Marchand P, Chargari C, Maroun P, et al.: Clinical outcomes of definitive chemoradiation followed by intracavitary pulsed-dose rate image-guided adaptive brachytherapy in locally advanced cervical cancer. Gynecol Oncol. 2015, 139:288-94. 10.1016/j.ygyno.2015.09.008

28. Gill BS, Kim H, Houser CJ, et al.: MRI-guided high-dose-rate intracavitary brachytherapy for treatment of cervical cancer: the University of Pittsburgh experience. Int J Radiat Oncol Biol Phys. 2015, 91:540-7. 10.1016/j.ijrobp.2014.10.053

29. Mahantshetty U, Krishnatry R, Hande V, et al.: Magnetic resonance image guided adaptive brachytherapy in locally advanced cervical cancer: an experience from a tertiary cancer center in a low and middle income countries setting. Int J Radiat Oncol Biol Phys. 2017, 99:608-17. 10.1016/j.ijrobp.2017.06.010

30. Pötter R, Georg P, Dimopoulos JC, et al.: Clinical outcome of protocol based image (MRI) guided adaptive brachytherapy combined with 3D conformal radiotherapy with or without chemotherapy in patients with locally advanced cervical cancer. Radiother Oncol. 2011, 100:116-23. 10.1016/j.radonc.2011.07.012

31. Rijkmans EC, Nout RA, Rutten IH, et al.: Improved survival of patients with cervical cancer treated with image-guided brachytherapy compared with conventional brachytherapy. Gynecol Oncol. 2014, 135:231-8. 10.1016/j.ygyno.2014.08.027

32. Wang F, Tang Q, Lv G, et al.: Comparison of computed tomography and magnetic resonance imaging in cervical cancer brachytherapy: a systematic review. Brachytherapy. 2017, 16:353-65. 10.1016/j.brachy.2016.11.001

33. Eifel PJ, Winter K, Morris M, et al.: Pelvic irradiation with concurrent chemotherapy versus pelvic and paraaortic irradiation for high-risk cervical cancer: an update of Radiation Therapy Oncology Group Trial (RTOG) 90-01. J Clin Oncol. 2004, 22:872-80. 10.1200/JCO.2004.07.197

34. Keys HM, Bundy BN, Stehman FB, et al.: Radiation therapy with and without extrafascial hysterectomy for bulky stage IB cervical carcinoma: a randomized trial of the Gynecologic Oncology Group. Gynecol Oncol. 2003, 89:343-53. 10.1016/s0090-8258(03)00173-2

35. Mayadev J, Viswanathan A, Liu Y, et al.: American Brachytherapy Task Group Report: a pooled analysis of clinical outcomes for high-dose-rate brachytherapy for cervical cancer. Brachytherapy. 2017, 16:22-43. 10.1016/j.brachy.2016.03.008

36. Rose PG, Ali S, Watkins E, Thigpen JT, Deppe G, Clarke-Pearson DL, Insalaco S: Long-term follow-up of a randomized trial comparing concurrent single agent cisplatin, cisplatin-based combination chemotherapy, or hydroxyurea during pelvic irradiation for locally advanced cervical cancer: a Gynecologic Oncology Group Study. J Clin Oncol. 2007, 25:2804-10. 10.1200/JCO.2006.09.4532

37. Whitney CW, Sause W, Bundy BN, et al.: Randomized comparison of fluorouracil plus cisplatin versus hydroxyurea as an adjunct to radiation therapy in stage IIB-IVA carcinoma of the cervix with negative paraaortic lymph nodes: a Gynecologic Oncology Group and Southwest Oncology Group study. J Clin Oncol. 1999, 17:1339-48. 10.1200/JCO.1999.17.5.1339

38. Rao BS, Das P, Subramanian BV, Jena A, Rashmi P, Konakalla VLA, Jayasree K: A comparative analysis of two different dose fractionation regimens of high dose rate intracavitary brachytherapy in treatment of carcinoma of uterine cervix: a prospective randomized study. J Clin Diagn Res. 2017, 11:XC06-10. 10.7860/JCDR/2017/22489.9607

39. Dimopoulos JC, Lang S, Kirisits C, et al.: Dose-volume histogram parameters and local tumor control in magnetic resonance image-guided cervical cancer brachytherapy. Int J Radiat Oncol Biol Phys. 2009, 75:5663. 10.1016/j.ijrobp.2008.10.033

40. Mazeron R, Fokdal LU, Kirchheiner K, et al.: Dose-volume effect relationships for late rectal morbidity in patients treated with chemoradiation and MRI-guided adaptive brachytherapy for locally advanced cervical cancer: results from the prospective multicenter EMBRACE study. Radiother Oncol. 2016, 120:412-9. 10.1016/j.radonc.2016.06.006

41. Pötter R, Dimopoulos J, Georg P, et al.: Clinical impact of MRI assisted dose volume adaptation and dose escalation in brachytherapy of locally advanced cervix cancer. Radiother Oncol. 2007, 83:148-55. 10.1016/j.radonc.2007.04.012 


\section{Cureus}

42. Bandera L, La Face B, Antonioli C, et al.: Survival and toxicity of radical radiotherapy (with or without brachytherapy) for FIGO stage I and II cervical cancer: a mono-institutional analysis. Eur J Gynaecol Oncol. 2014, 35:121-7.

43. Georg P, Pötter R, Georg D, et al.: Dose effect relationship for late side effects of the rectum and urinary bladder in magnetic resonance image-guided adaptive cervix cancer brachytherapy. Int J Radiat Oncol Biol Phys. 2012, 82:653-7. 10.1016/j.ijrobp.2010.12.029

44. Kirchheiner K, Nout RA, Lindegaard JC, et al.: Dose-effect relationship and risk factors for vaginal stenosis after definitive radio(chemo)therapy with image-guided brachytherapy for locally advanced cervical cancer in the EMBRACE study. Radiother Oncol. 2016, 118:160-6. 10.1016/j.radonc.2015.12.025

45. Spampinato S, Fokdal LU, Pötter R, et al.: Risk factors and dose-effects for bladder fistula, bleeding and cystitis after radiotherapy with imaged-guided adaptive brachytherapy for cervical cancer: an EMBRACE analysis. Radiother Oncol. 2021, 158:312-20. 10.1016/j.radonc.2021.01.019 